\title{
Elementos que fundamentan y guían el conocimiento tradicional de los saberes médicos de los afrocolombianos e indígenas de Pizarro, Chocó
}

\section{Elements that base and guide the traditional knowledge of the medical knowledge of the Afrocolombian and indigenous of Pizarro, Chocó}

\author{
Jesús Dante Mosquera Orejuela*
}

\section{RESUMEN}

Los habitantes afros e indígenas del municipio de Pizarro, han conservado sus patrones culturales y los conceptos de sus ancestros logrando la continuidad de diferentes saberes que el hombre de esta región aprendió a conservar para su propia supervivencia. Este trabajo buscó identificar los principales elementos que guían el conocimiento tradicional de los saberes médicos o terapéuticos de la zona. Para ello, se realizó una revisión crítica de la información bibliográfica sobre la cultura de sus poblaciones, así como diálogos con los conocedores de las diferentes terapias empleadas. También se realizaron observaciones directas, recorridos y visitas espontáneas y planificadas, entrevistas abiertas o estructuradas, elaboración de diario de campo y talleres, que permitieron identificar cuatro paradigmas o elementos que guían el conocimiento tradicional de los saberes médicos: el equilibrio psicológico-mágicoreligioso, las siete enfermedades inherentes al ser humano, el hecho de que toda planta sirve para curar y el poder de lo frío y lo caliente. Asimismo, se lograron identificar sus principales técnicas semiológicas, terapéuticas, mágicas y de brujería. La información levantada permitió concluir acerca de la importancia de las «cabezas médicas» afrodescendientes e indígenas dentro del mantenimiento de la salud, calidad de vida de las comunidades y la sostenibilidad del conocimiento tradicional.

Palabras clave: Afrodescendientes; Conocimiento tradicional; Indígenas; Saberes.

\begin{abstract}
The Afros and Indigenous inhabitants of the municipality of Pizarro, have conserved their cultural landlords and the concepts of their ancestors obtaining the different continuity of knowledge that the man of this region learned to conserve for his own survival. The present work looked for to identify the main elements that guide the traditional knowledge of therapeutic doctors or of the zone. For it was realized a critical revision of the bibliographical information on the culture of his populations, as well as dialogues with the connoisseurs of the different used therapies. Additionally direct observations, routes and spontaneous visits were realized and planned, open or structured interviews, field newspaper elaboration and factories, which allowed to identify four paradigms or elements that guide the traditional knowledge of knowledge doctors, the balance psychological-magician-monk, the seven inherent diseases to the human being, the fact that all plant serves to cure and the power of it cold warms up and it. Also they were managed to identify his main semiologyc, therapeutic, magical techniques and of witchcraft. The raised information
\end{abstract}

\author{
Antropólogo. Docente Universidad \\ Tecnológica del Chocó «Diego Luis \\ Córdoba», Quibdó, Colombia. \\ e-mail:americosa29@yahoo.es \\ Recibido: 10 de marzo de 2011 \\ Aceptado: 20 de abril de 2011
}




\section{Bioetnia Volumen 8 No 1 (enero-junio), 2011}

allowed concluding about the importance of the Afrocolombian and Indigenous medical heads within the maintenance of the health, quality of life of the communities and the sustainability of the traditional knowledge.

Keywords: Medical heads; Traditional knowledge; Indigenous and Afro-descent.

\section{INTRODUCCIÓN}

Todos los seres humanos, gracias a su naturaleza biológica, su cerebro y sus estructuras mentales, independientemente de su entorno cultural, poseen la capacidad de producir procesos de abstracción de su realidad y emiten proposiciones demostrativas como formas particulares de saberes o conocimiento. El desarrollo de las ciencias humanas permitió que se dejara de mirar al hombre como un objeto biológico de estudio, y más bien se lo entendiera como un sujeto de saberes con una extraña capacidad de poderes, para representar la vida y los discursos de distintas maneras. Que se mirara al hombre como un hacedor de culturas diferentes, con filosofías regionales, epistemologías locales y lenguajes particulares, siendo estas nuevas posibilidades las que permiten la existencia de otras formas de saberes.

Gracias a ello, las sociedades tradicionales pueden explicar los fenómenos de las cosas desde su génesis u originalidad interrelacionándolos con ideas cosmogónicas, sobrenaturales y religiosas, dándole así un carácter material, espiritual y divino a todos los elementos de la naturaleza y la cultura, las que actúan gracias a la fuerza que en ellos imprimen los dioses y los espíritus. Son estas las razones que motivaron la realización de este estudio relacionado con los elementos que fundamentan y guían el conocimiento tradicional de los saberes médicos y terapéuticos de afrocolombianos y amerindios de Pizarro. Estos elementos están relacionados con el conocimiento tradicional que tanto unos como otros vienen aplicando como mecanismos de supervivencia, basados única y específicamente en la convivencia que a través del proceso de imbridación que les dio la vida en el entendimiento de hombre y naturaleza, lograron permear un desarrollo existencial, en el que el hombre de esta región se identifica con su medio natural y el medio natural le proporciona los recursos necesarios para la continuidad de su existencia.

No obstante, es válido reconocer que aun desconociendo otros saberes como la medicina facultativa, la conceptualización de la medicina ayurvédica (conocimiento de las plantas, procesos mágico religiosos, cantos del jai, embrujamientos y tantos otros subterfugios utilizados por los hombres de esta región), ha logrado sostener sus condiciones de salubridad, por encima de sus condiciones de vida, razón por la cual, los indígenas y afrodescendientes del Chocó, en especial en Pizarro, poseen grandes elementos y conocimientos en la medicina tradicional para superar el incumplimiento de las políticas estatales. Estos elementos se materializan también en la sostenibilidad de conceptualizaciones tales como hacer el bien o hacer el mal, utilizando complejos de difícil entendimiento como: «la madre de agua, el tamborito, la cogida del rastro, los famosos procesos del mancua para enamorar, los salamientos de las casas y personas, la veeduría de la suerte, los encantamientos, las bebidas ontogénicas» y muchísimas otras expresiones y manifestaciones de la vida de este conocimiento tradicional que ha sostenido la vida sociocultural de los pueblos no solo de Pizarro sino del Chocó biogeográfico y pluricultural.

Esta investigación propende por construir una línea base de información, del conocimiento tradicional terapéutico de las comunidades amerindias y afrodescendientes del Chocó biogeográfico, que permita el diseño de estrategias, mecanismos e instrumentos que protejan tanto el objeto del conocimiento, como al sabio nativo. Esto implica la protección del entorno ambiental y las condiciones de creación, transformación y socialización de los saberes de tan importantes personajes y los acumulados de los cuales son portadores y guardianes. En síntesis, se trata de contribuir mediante la documentación de la información, los procesos de erosión de la etnicidad, buscando mantener la unidad dialéctica de lo tangible y lo intangible del saber.

Elobjetivo de este artículo es realizar una etnografía de los principales elementos que guían el conocimiento tradicional de los saberes médicos o terapéuticos de los afrocolombianos y amerindios de Pizarro en el Chocó.

Los objetivos específicos son: identificar los principios que rigen el sistema terapéutico tradicional de los grupos afrocolombianos e indígenas que habitan el territorio de Pizarro en el departamento del Chocó y describir la caracterización de las «cabezas médicas» que guían la terapéutica tradicional de afrocolombianos e indígenas en Pizarro y algunos elementos del sincretismo cultural.

\section{MARCO CONCEPTUAL}

Antropología médica. La antropología médica es vista como una subdisciplina biocultural, que concierne a aspectos biológicos y culturales del componente humano, dado que en la historia humana se ve influenciada en la salud y la enfermedad. La naturaleza biocultural de la antropología médica se encuentra latente en una serie de reseñas críticas que aparecieron algunos años atrás. Portela (1998) hace una síntesis de los inicios de la antropología médica, partiendo de Rivers (1924) hasta los estudios de Aguirre (1946), con que se despejan muchas incógnitas.

- La medicina tradicional es integral, la medicina moderna solo tiene en cuenta la enfermedad.

- En los pueblos tradicionales se observa una dicotomía de 
las enfermedades en dos categorías, enfermedades que el médico puede curar y enfermedades «populares» que los médicos se niegan a reconocer.

- Los sanadores tradicionales son de edad avanzada, muy respetados en todas las comunidades y deberían ser aliados en los programas de atención primaria.

- Los médicos que trabajan en «medios tradicionales» ignoran con frecuencia la medicina tradicional y tienen problemas de comunicación con sus pacientes.

Foster y Anderson (1978) consideran que cada sistema médico abarca todas las creencias que promueven la salud, las acciones y el comportamiento científico y la habilidad de los miembros del grupo que los suscriben. Estos autores distinguen un sistema médico dado en dos partes: el de teoría de enfermedad y el de cuidado o atención de la salud. El primero, reconoce las creencias acerca de la naturaleza de la salud, la causa de la enfermedad, las cualidades de los remedios y técnicas utilizadas para contrarrestarla.

A nivel nacional sobresalen las investigaciones hechas por los esposos Reichel-Dolmatoff, (1979); importantes aportes son los de Gutiérrez (1985) sobre la medicina tradicional, como también los realizados por Herrera y Loboguerrero (1982).

A nivel local, el trabajo investigativo encontró que Velásquez (1957) en «Muestras de fórmulas médicas utilizadas en el Alto y Bajo Baudó», hace una clasificación de las enfermedades en el ámbito de edad y sexo, y se dan las diferentes fórmulas utilizadas para curarlas.

Este panorama histórico del desarrollo de la antropología médica desde la perspectiva de la etnomedicina, permitió fundamentar las bases teóricas para elaborar el trabajo de investigación enunciado. La casi total inexistencia de fuentes de referencia en el departamento del Chocó, así como las muy escasas investigaciones realizadas al respecto de las «cabezas médicas» en la región, las dificultades de acceso y la dispersión de los asentamientos de amerindios y afrodescendientes o sus entornos entre otras tantas circunstancias insalvables, ameritaron hacer uso de estos postulados teóricos de conceptos metodológicos. Como el desconocimiento de las prácticas tradicionales de los habitantes de esta diada (indios y negros como se les señala para la exclusión), más bien ha sido juzgado en lugar de ser estudiado, pues el mundo de occidente no reconocía ni valoraba lo que ellos no consideraban como ciencia y estas prácticas tradicionales no formaban parte del episteme de estos «sabios», sin embargo han sido valiosos la contribución y el aporte tanto de indígenas como de afrodescendientes. En consecuencia, la investigación estará referida a identificar las «cabezas médicas» y analizar la funcionalidad de los sistemas médicos tradicionales con que el hombre de la región ha logrado sobrevivir, teniendo en cuenta las condiciones de marginalidad y de beneficios con que puede aportar para mejorar el sistema de salud terapéutico y facultativo, si se produjera una hibridación de los dos sistemas.

El Chocó es uno de los departamentos más deprimidos de Colombia en cuya construcción histórica, sus habitantes se han venido «peleando» la solución a sus necesidades básicas o primarias frente a la institucionalidad local, departamental y nacional como es el caso de la salud, para poder mitigar las epidemias, las endemias y las pandemias que han afectado y afectan sus formas de vida. La triada de pobladores: indígenas, afrodescendientes y mestizos, carecen del bien más preciado que posibilita la existencia del ser humano, cual es la posibilidad de existir en condiciones de bienestar que permitan su proyección humana.

La antropología socio-cultural ha descubierto que el primer aspecto de equilibrio que presenta el ser humano, es el que se refiere al estado de salud del hombre. Mediante la salud, el Homo Sapiens Sapiens dispone psicológicamente sus capacidades y aptitudes para cumplir no solo funciones corporales, sino también desarrollar sus preocupaciones mentales. Por tanto, podemos colegir que el centro de equilibrio que permite la función activa de vida del ser humano es la salud, que involucra los diferentes procesos que el hombre elabora en sus distintos hábitats y con los recursos que le provee la naturaleza ambiental, construye y elabora fórmulas para asegurarse la continuidad de sus días.

El chocoano común posee un condicionamiento cultural múltiple en la atención de la salud y participa prácticamente en todos los sistemas existentes. Cuando enfrenta la enfermedad, busca su solución, primero en el ambiente inmediato, el hogar, con la medicina casera y continúa recurriendo a los demás sistemas médicos. Está preparado para que cada tipo de medicina pueda darle una respuesta condicionada a sus expectativas y adecuada a su trasfondo cultural y así se mueve a plenitud dentro de cada uno. Las «cabezas médicas» ubicadas a lo largo y ancho del Chocó y en sus 31 municipios, operan según las circunstancias causales y las reacciones culturales. De allí se desprenden diversos sistemas en la aplicación de la medicina tradicional, a saber:

El sistema mágico florece cuando la estructura institucional es incapaz o está distante para dar respuesta efectiva y pronta a sus reclamos y conflictos. Gutiérrez (1985) afirma que «el sistema mágico sustituye la acción legal; es paliativo en la emergencia económica; reemplaza el apoyo religioso; da respuesta psico-afectiva gratificante y salida a las tensiones que se generan en las redes sociales que rodean al individuo y las resuelve ajustándose a los patrones culturales de relación de los egos.

El sistema mágico-religioso satisface conflictos éticos internos de la fe cristiana con amplitud. Mediante la absolución o la bendición, reivindica al pecador enfermo, lo exonera de culpa, lo exalta y le da apoyo espiritual para el alcance de bienestar o para aceptar en forma más ajustada y sostenida 


\section{Bioetnia Volumen 8 No 1 (enero-junio), 2011}

por la fe, el mal que padece.

La medicina casera. En cualquier sistema médico esta resuelve distintos interrogantes en el ámbito social, por ser naturalística o afianzada en lo sobrenatural. Su praxis y principios teóricos se insertan en los distintos niveles tecnológicos y científicos del acontecer médico. Sirve de paliativo o de prueba, mientras se constata la gravedad de la dolencia y se definen los pasos a seguir en la aceptación del rol de enfermo o de paciente.

El curanderismo es el que más se relaciona con el facultativo, de alguna manera sirve a los estamentos sociales. Sus principios teóricos y prácticos se acoplan al nivel tecnocientífico de cada enfermo, dando respuesta positiva a su ansiedad. En estas circunstancias el uso de la farmacopea vegetal y de sustancias médicas animales y aun humanas, se mantiene con una amplia difusión para subsanar cualquier emergencia de salud.

El concepto de enfermedades culturales en los sistemas médicos tradicionales es otra razón que explica su permanencia y funcionalidad. Este principio teórico ha mantenido vivas y actuantes las enfermedades culturales, que se asocian generalmente a endemias regionales o a grupos de edad, dado que cualquier «cabeza médica» tiene la facultad de dirimir la naturaleza y en uno u otro sistema, pueden llegar a la consulta pacientes con auto diagnóstico de enfermedad natural y resultar clasificados como pacientes mágicos o a la inversa. La academia y con ella el mundo occidental y occidentalizado, hace muy poco tiempo empezaron a darle valor a los saberes no académicos, producidos por vías de lógicas no cartesianas. Las principales víctimas de estas prácticas han sido históricamente los grupos étnicos, en particular los distintos a los europeos.

Algunos elementos geográficos y etnográficos de las poblaciones del municipio de Pizarro, Chocó. Las tierras bajas del Pacífico son un fenómeno geomorfológico que históricamente ha llamado la atención de humanistas y ante todo, de los especialistas en ciencias de la tierra. Colombia ocupa un territorio de gran dimensión conocido como Andén Pacifico, región comprendida entre la cordillera del Darién y el golfo de Urabá al norte, al oriente la cordillera occidental, al sur la república de Ecuador y al oriente el océano Pacífico». En este convergen diversos grupos humanos, de los cuales solo se hace referencia políticamente a los que corresponden a Pizarro, Chocó:

Los paisas. Se hacen presentes en el Chocó en volúmenes significativos después de 1940 con la apertura de la carretera Quibdó-Medellín. Representan admiración por su imagen y empuje, y están diseminados por todo el Chocó. Son «rebuscadores» y hacen lo imposible por «conseguir» lo que buscan.

Anglosajones. Dejaron su impronta en el Chocó a finales del siglo XIX, cuando irrumpieron con la minería industrial.
Hacia la segunda década del siglo XX, fueron reemplazados por los estadounidenses, en la misma actividad económica.

Españoles. Su presencia en tanto volumen de gentes y su condición de metrópoli colonial dejó sellos indelebles en la cultura afrochocoana: el idioma, el judeocristianismo, la tradición leguleya y muchas otras prácticas de su etnicidad.

Franceses. Penetraron a la élite intelectual y económica, desde la guerra de Independencia hasta las primeras décadas del siglo XX. Su sello está en el lenguaje, literatura, prácticas culturales y modales.

Los indígenas. Como categoría colonial y como fenómeno social, se han prolongado mucho más allá de la llamada Independencia, pues las condiciones económicas y las diversas presiones y mecanismos que los rodearon originalmente, han perdurado en su esencia aunque con ligeras modificaciones. En el Chocó, donde antes eran clasificados como caribes, parecen ser una familia independiente, conformada por los Chamí, Catíos o Eperará, que viven principalmente en el Pacífico, siendo los principales grupos:

- Los Waunana. Como segunda familia indígena de este departamento, se ha convertido en un verdadero «quebradero de cabeza» entre los etnólogos, pues algunos la clasifican como un grupo étnico particular y otros la ubican como un subgrupo de los Embera.

- Los Embera. Son el grupo mayoritario, que a su vez se divide en dos grandes troncos: los Embera de montaña o eyávidas y los Embera de río o dódivas. A los Embera la literatura etnográfica los conoció como Chamíes o Katíos. Los nuevos nombres son autoetnónimos y no accidentes geográficos como se les denominaba.

Los afrodescendientes. A juzgar por determinados usos culturales y la variedad dialectal, se confirman las pesquisas de los etnólogos, cuando afirman la existencia de la presencia viva de etnicidades de los grandes grupos culturales africanos, a despecho de los documentos paleográficos relacionados con los grupos étnicos transportados a América, porque estos fueron alterados en su origen por razones diversas.

Algunas definiciones dentro del conocimiento tradicional sólo hay una

Medicina popular y/o medicina tradicional. Son tradiciones terapéuticas que integran lo que se denomina el modelo alternativo de salud, donde se agrupan todos los saberes médicos que no se encuentran en el modelo médico hegemónico occidental.

\section{DISEÑO METODOLÓGICO Y TRABAJO DE CAMPO}

La metodología de investigación utilizada incluyó:

Revisión crítica de la información bibliográfica sobre poblaciones afrocolombianas y amerindias de Pizarro.

Definición de actividades y procedimientos necesarios para recolección de información de campo sobre aspectos 
centrales de la vida de la comunidad, en especial a su relación con el medio y los recursos naturales.

- Diálogo de saberes tradicionales con los distintos conocedores de las diferentes terapéuticas de los problemas que afectan a los habitantes de la región.

- Definición y aplicación de técnicas de obtención y registro de información cualitativa, que incluye, además de los formatos diseñados, observación directa, observación participante, recorridos y visitas espontáneas y planificadas, entrevistas abiertas, entrevistas estructuradas, elaboración de diario de campo, grabación de testimonios orales, registros fotográficos y talleres.

La investigación se realizó específicamente en el municipio de Pizarro (Bajo Baudó), entre los pueblos de San Agustín de Terrón, Cuevitas, Virudó, Orpúa, Belén de Docampadó, Pavasa, Pilizá, Usaragá, Sibirú y Puerto Meluk. No obstante, las manifestaciones que aquí se expresan, se presentan también en los otros municipios chocoanos con mayor o menor frecuencia entre los pobladores indígenas y afrodescendientes.

\section{RESULTADOSYDISCUSIÓN}

Principios organizadores de la etnomedicina del municipio de Pizarro. La etnomedicina o sistemas terapéuticos tradicionales del Chocó biogeográfico es un concepto que integra diversos paradigmas de salud a saber: el equilibrio psicológico-mágico-religioso, filosófico y económico. Un segundo paradigma lo constituyen las enfermedades latentes inherentes a todos los humanos, es decir, el estado latente, potencial, que permanece en el organismo hasta que una causa cualquiera los despierta, ellas son «las siete enfermedades», que todo individuo trae al nacer, de las que tifo o tabardillo, sífilis, lombrices y tuberculosis son las principales. El tercer paradigma es: «Toda planta sirve para curar», siguiendo la «cabeza médica». En este sentido, se advierte que en la terapéutica tradicional la farmacopea y la farmacognosia son indispensables, sin olvidar el empleo de otras sustancias. Lo frío y lo caliente constituyen el cuarto paradigma del análisis realizado en la investigación, como la clave del diagnóstico y la terapéutica.

Desde esta óptica se desprenden en las concepciones de los afrodescendientes e indígenas de la zona, con sus distintas técnicas semiológicas en el proceso de curación (sanar) o del bien, ofender (agredir) hacer el mal; a continuación se describe cómo funcionan estos paradigmas:

Técnicas semiológicas. Lo primero que debe determinar la «cabeza médica» en su diagnóstico es si la enfermedad es natural de Dios o es accidental del diablo, para ello recurre a una inspección ocular, luego a un detallado interrogatorio a los familiares del paciente, haciendo una inspección sigilosa de los sitios que frecuenta el enfermo. Una detenida inspec- ción de la visita del consultante ayudará a concluir si el achaque es causado por «mal pecho», si es «puesto», etc, en caso negativo se procede a actuar con la impresión diagnóstica de una enfermedad natural o accidental. Pero antes «se lee la orina», la cual, según el olor, color, sabor y las diferentes sedimentaciones establece: diagnóstico de certeza o pronóstico probable. A posteriori la «cabeza médica» se centra en los síntomas y signos del afectado: vómitos, hipertermia, hipotermia, respiración profunda, pulso acelerado, diarreas, baba pastosa, mal aliento, hipertermia tópica, mareos, nauseas, etc., que van orientándolo hacia el diagnóstico. Solo después aparecen pruebas locomotrices, percusiones, palpaciones, audiciones de pulmones, vas digestivas, etc.

Si la enfermedad esta encubierta, se acude a suministrarle un caldo de jurel (pez marino) que hace brotar las venéreas o infecciones «tapadas», la carne de león (macho) es una ayuda para hacer brotar por la piel los males ocultos. Cuando la enfermedad es de Dios, se acude a un especialísimo vademécum de oraciones, invocaciones, secretos, etc. Si no hay pronta mejoría, se sabe que es Satán quien actúa. El maléfico se contrarresta con diversas alternativas mágicas como las oraciones, talismanes, contras y cuando es menester, se lucha contra el propio Satán. Sobra decir que a esto último solo se le miden los chinangos muy poderosos: «Yo soy el dueño de los placeres, ¿quién me llama?» Inmediatamente empieza a desarrollarse el compromiso o intercambio, pues el interesado debe firmar un pacto de sangre con el diablo quien le pide su alma o el alma de sus seres queridos.

Técnicas terapéuticas. Elaborado el diagnóstico de certeza, si la enfermedad es de Dios, se apela a un catálogo altamente especializado de oraciones. Si al cabo de unos días el paciente empeora, se infiere que es una enfermedad del maligno. Entonces el chinango debe hacer gala de toda su sapiencia para realizar los desentuertos, destramo, etc., debiendo acertar la contra que bloquee el ardid empleado por otro chinango que se apoyó en el diablo. En esta terapia intervienen los contrasecretos, minerales, animales, partes de animales, yerbas, reliquias de santos y productos forestales. Cuando se trata de una enfermedad natural, la voz cantante corresponde a la herbolaria y en menor medida a otros productos; en caso de accidentes como fracturas, intoxicaciones, etc. el médico tradicional actúa similar a su colega alópata, diferenciándose en que además se vale de oraciones mágicas, secretos que pegan huesos, sacan humores, etc.

Magia y medicina. Son dos actividades virtualmente inseparables. La «cabeza médica» basa su estrategia terapéutica en su habilidad para manipular la naturaleza y el mundo espiritual, a fin de lograr el propósito deseado; siempre antes de actuar se encomienda a deidades protectoras. Los «materiales» empleados liberan sus energías o principios activos después de pedir autorización expresa a las deidades tutelares, para que las virtudes de las plantas y los productos 


\section{Bioetnia Volumen 8 No 1 (enero-junio), 2011}

farmacognósticos y farmacopéicos cumplan su cometido. Así pues, el facultativo, ya sea formado por maestros o iluminados (aquellos a quienes un ser suprahumano, en un estado alterado de conciencia: sueños, pesadillas o en contacto directo, les ha revelado el «conocimiento») está facultado para intervenir cambiando el rumbo de los acontecimientos mediante sus poderes, secretos y saberes de «materiales».

La efectividad terapéutica está condicionada a que tanto pacientes como familiares y el médico hayan «dormido» bien, sobre todo, cuando este procede a beneficiar las plantas medicinales. Es decir, no haya tenido relaciones sexuales recientemente, las mujeres no estén menstruando o no hayan parido en los últimos días. Es preciso neutralizar el «mal pecho» (bajas pasiones, rencores, envidias, ambiciones, celos, etc.) que desvían la energía positiva, o hacen que la labor benéfica se neutralice.

Concepto de lo frío y lo caliente. Bajo este pensar se fundamenta el tratamiento de las enfermedades en la zona de estudio. Por la búsqueda del equilibrio ideal, frío y caliente se contraponen, mas en unidad dialéctica van unidos inseparablemente. Si el organismo se descompensa por hipotermia, la base de la terapia apunta a equilibrarlo con una carga contraria. Si el caso es opuesto, para lograr la equidad se acude a remedios y alimentos que bajan la temperatura. En consecuencia, todo estado de alteración de la temperatura es un síntoma que indica enfermedad. Ello llevó a estos sabios a establecer una clasificación de enfermedades frías y calientes, y por ende, remedios ídem. Algunos ejemplos son: «Con excepción de dientes y muelas, las enfermedades calientes abarcan de la cabeza a la cintura.» A este conjunto pertenecen las venéreas de fuego y las retenciones urinarias, el reumatismo de calor y la fiebre palúdica, el bazo y el hígado, el mal «de corazón»y los calores estomacales, algunas enfermedades de la sangre y la tisis galopante, la ceguera, el tabardillo y la ictericia; ciertas infecciones del riñón, la tosferina y las hernias, el sarampión, las viruelas, la bronquitis, el colerín, las gripas y el asma o ahoguín, los dolores estomacales, de la cabeza y la debilidad de los pulmones.

Además de las citadas son propias de la niñez, las lombrices y los espantos de tierra, mal de ojo y los sapitos o afta, las perlas o roséolas. Para muchos curanderos los llamados espantos de tierra y agua, y el mal de ojo, son enfermedades nerviosas causadas por la envidia. Con estos achaques quedan prohibidos los alimentos calientes, al menos que, por el temple y constitución del paciente, la enfermedad haya evolucionado a fría o se desee practicar la sentencia «similar similibus curantur» (lo similar cura).

Referente a las enfermedades opuestas, se obtiene el siguiente marco:

Enfermedades frías. Los quebrantos fríos aparecen de la cintura para abajo. Toman su asiento en estas partes del cuerpo por ser las extremidades inferiores las que más perma- necen en contacto con la tierra, el agua, las piedras y las sustancias minerales consideradas frías en el concierto regional. Con todo, algunas toses, caries y abscesos dentales, debilidades pulmonares, del riñón, convulsiones de los recién nacidos, pican y tétanos de las heridas, pican y sus secuelas, caquexias y ulceras incurables, para citar algunas, se ubican en este grupo, no porque aparezcan de la cabeza para abajo, sino por ser producidas por agentes fríos, tal es el caso de las bubas originadas por las succiones de los murciélagos en el cuerpo de los campesinos. El murciélago, se dice es frío por su trabajo nocturno.

Son remedios fríos el agua común hervida, azúcar, leche, ají dulce, manzanilla, linaza, yuca, coco, col, plátano guineo, banano primitivo, papa, bacao, verdolaga, escoba babosa, es cancel, limoncillo, toronjil, caracucho blanco, sábila, saúco de monte alto, sal de Epson, jengibre, clara de huevo, arroz, aceite, camine, queso, huevo, cebolla en rama, venturosa, enviande, malva, siempreviva, hierba mora, escobilla, espadilla, grama, cebada, caledonia, caña de azúcar, caña agria, las verduras y las frutas. Entre los animales: carne de raía, tortuga, sapo, gallina, caimán, lagarto, gusano, ardilla, araña, guatín, comején, avispa de tierra, baba de anguila, lombriz de seres humanos, leche de vaca y de mujer bien negra. Entre los minerales: piedra y arena de río, barro blanco, negro y colorado, alumbre, joyas de oro y plata.

Elementos médicos calientes. En la etnomedicina chocoana de las «cabezas médicas», aparece un listado de medicamentos calientes, como: cebolla de cabeza, yerba de sapo, amargo andrés, ajenjo, ajonjolí, nacedero, sauco, quina, ortiga, maíz, paico, poleo, ají, guayaba, ruda, cilantro, santa maría, doña juana, boba, algodón, lulo, friega platos, albahaca, guamo, Marín Gálvez, apio, verbena negra, verbena morada, chillaran morado, tabaco, plátano, gallinacito, canchalagua, rosa, yerbabuena, menta, san Juanito, gualanday, chiva, palosanto, zaragoza, miel de abejas, sen, sal común, aceite de castor, aceite de comer, aceite de almendras, manteca de corozo, brea, flor de azufre, canela, comino, nuez moscada, pimienta dulce, pimienta picante, café amargo, ajo, chocolate, pan tostado, clavo, aceite, caraño, aceite canime, quina, hielo, ceniza, esencia de menta, esencia de vainilla, bálsamo de copaiba, romero, alhucema, borraja, agua de mar, cascarilla, bálsamo de Tolú, bálsamo de bongué, anís, alcohol, sal de Inglaterra, vino seco, aguardiente, aguardiente de Alemania, agua florida, bay rum, tónico Bayer, permanganato, pólvora, coirón, paludismo, píldoras de vida, vigorón y muchos otros productos patentados. Se identifican con este grupo: manteca de petacona o sobrecama de oídos o de armadillo, de lombriz y de zorra, de león, lagarto, caimán, nupa, gallina, res y cerdo; cueros de venado, perico y cuzumbí o cozumo y vaca; pezuña de la gran bestia o tapir americano entre otros.Entre los remedios indeterminados aparecen los de procedencia humana como uñas, saliva, cordón umbilical, orina, sudor, etc. 
La Cábala y la numerología. En el oculto universo del Chocó profundo, se encuentran continuos culturales africanos en pleno vigor. Por ejemplo, los mellizos llenan de fortuna el hogar de donde son producto. Padre y madre, son reputados por su virilidad y fecundidad, el uno posee la fuerza y el otro la habilidad. Uno inteligencia, otra intuición y lo que sufre el uno lo siente el otro, son pues como un solo ser dividido en dos.

Sobre la brujería. Esta es la parte más oculta de estos saberes, es exclusiva de oficiantes o iniciadores que han accedido a ciertos secretos. La idea fundamental consiste en manipular la naturaleza a través de fuerzas maléficas o benéficas, concentradas en la mente; la naturaleza por medio de sus productos y seres suprahumanos, con oraciones, reliquias, entuertos, produce alteraciones en el equilibrio de las personas objetos. Así por ejemplo, para conquistar amores, se toma una hebra de cabello de la agraciada, se introduce en el filtro amoroso, con pájaro mancuá, quereme y sígueme los pasos. Después de rezada la oración, al poco tiempo la dama vendrá rendida a los pies del interesado.

Existen magias para matar a enemigos, en esta práctica se hace un muñeco parecido a la víctima o se consigue una foto, se introduce en un vaso con agua, el retrato ira invertido, se le encienden siete velas amarradas con una cinta negra. Se le pone la oración por siete días, previo entierro del macuto (muñeco). El enemigo perderá lentamente sus energías hasta morir. La madre de agua, la cogida del rastro, la trampa de parturienta, etc., hacen parte de este repertorio, al igual que las ombligadas y otras tantas argucias que entretejen la sabiduría de indígenas y afrodescendientes de la zona.

En algunas fórmulas médicas cristianas para destramar a una parturienta se invoca a la intervención de San Francisco con esta oración: «el Señor te guarde y te bendiga y vuelva a ti su rostro. El Señor haga de ti misericordia y te de paz a ti, $\mathrm{NN}$, de tu santa bendición». En la magia parturienta, tramada: se calienta un limón que se hace acompañar entre los acompañantes de la paciente. El sortilegio se va debilitando cuando pasa de mano en mano, a fin de no quemarse los participantes. Así dará a luz la criatura. Un ensayo homeostático: «Por el camino del ciprés, con Jesucristo caminé, con San Pedro y San Pablo, San Juan y San Miguel. Como se detuvo nuestro Señor Jesucristo en el santo árbol de la cruz, detengo esta sangre; como se detuvo nuestro Señor Jesucristo en la sagrada hostia, detente sangre; como se detuvo la preciosísima imagen de Nuestro Señor Jesucristo en el mundo, para bien de los pecadores, detente sangre; como se detuvo Nuestro Señor Jesucristo sobre sí mismo, amén», se rezan tres credos.

Diferencias entre saberes afrodescendientes e indígenas. En este sentido debemos reconocer la diferencia histórica que se establece entre los indígenas y los afrodescendientes, por cuanto en primera instancia los primeros pobladores del municipio de Pizarro, fueron los indígenas, quienes con sus conocimientos y saberes ancestrales implementaron formas de vida que posteriormente compartieron con nuevos habitantes que no eran de su génesis racial. Seguidamente los invasores europeos intervinieron los procesos socioculturales que los nativos de la región tenían como modelos de supervivencia étnica, incluyendo a los africanos en el territorio. De tal manera, se conformó el advenimiento de saberes médicos que sustentaron la sostenibilidad de lo ancestral y lo ayurvédico con lo facultativo.

La herencia indígena identificada en el Chocó, presenta un sistema mágico-religioso asociado con prácticas curativas de farmacopea vegetal, mediante la conquista de recursos del entorno físico. Asimismo, los afrodescendientes con el conocimiento de los factores del medio ambiente y dotado de «poderes» mágico positivos y de agresión, tras un largo proceso formativo, causan la enfermedad y la cura y ambas etnias mediante rituales y ayudados por alucinógenos alcanzan un trance chamánico que los introduce al mundo de los espíritus, de lo mítico y de los recursos culturales, el mensaje de curación o de agravio a sus pacientes. El poder mágico escapa del campo médico para introducirse al poder de la adivinación, configurando una personalidad líder, «todopoderosa» dentro de la comunidad.

El español transmitió un sistema médico oficial, el de la medicina aculturativa, suma de legados antiguos pero coartado por principios escolásticos, que desconocían el ecosistema americano en sus factores positivos y agresivos en torno de la salud, y su sistema médico no cubría las exigencias del hábitat. En forma encubierta las comunidades vivieron y transmitieron, una medicina mágico religiosa, que se expresaba en oraciones, en salmos, jaculatorias, invocaciones, exorcismos y prácticas curativas, agresivas y preventivas, que fueron especialmente asimiladas por los afrodescendientes.

Los afros llegan culturalmente desintegrados. Aun cuando también trajeron un subtratum mágico y un sistema médico, que no pudo proyectar abiertamente, como unidad rota que era, en un ambiente físico diferente y en una comunidad antagónica. Su mentalidad mágica le sirvió para captar y asimilar la española e indígena, un proceso fácil de aculturación. De ello se desprende que hoy existan y se perfilen dos versiones de la medicina no solo en el Chocó, sino también en todo el país colombiano: una orientada por la academia y otra definida por el saber y la práctica popular, medicina facultativa y medicina tradicional impulsada por las «cabezas médicas», que ejercen sabiduría a todo lo largo y ancho del país.

Lo más significativo de estas diferencias estriba en que los indígenas en sus procesos de curación se apoyan específicamente en el canto del jai (proceso mediante el cual invocan la presencia y ayuda de poderes sobrenaturales, en espacios sutilmente decorados con plantas y palmas, tinturas, baños 


\section{Bioetnia Volumen 8 No 1 (enero-junio), 2011}

y el permanente consumo de bebidas embriagantes: chicha, guarapo, biche, entre otros). Los negros en cambio (trabajan el proceso curativo y agresivo), a través del conocimiento de las plantas, oraciones y la invocación de duendes, animales y demonios así como del panteón de distintos santos y vírgenes que son sus «ayudadores».

Caracterización de las «cabezas médicas» que guían la terapéutica tradicional de afrocolombianos $e$ indígenas. La sostenibilidad del conocimiento tradicional fue logrado históricamente por las «cabezas médicas» existentes antes y después de la llegada de los conquistadores. Es así como en el Chocó y en el entorno colombiano, terminan por perfilarse dos grandes versiones de la medicina: una orientada por la academia y otra orientada por el saber y la práctica popular: medicina facultativa y medicina popular o tradicional. Aquí se conjugan la herencia india y la herencia negra identificadas por un sistema mágico-religioso asociado con prácticas curativas de farmacopea vegetal, mediante la conquista del entorno físico y el conocimiento de los factores agresivos del medioambiente y de algunos sistemas para contrarrestarlos.

Las «cabezas médicas» «dotadas» de poderes mágicos positivos y agresivos, tras un largo proceso formativo, causan la enfermedad y la cura. Mediante rituales y ayudados por alucinógenos, alcanzan en trance chamánico la introducción al mundo de los espíritus. De lo mítico y con los recursos naturales, de manera consciente aportan el mensaje de curación o de agresión de sus prácticas.

El poder mágico escapa del poder médico facultativo y se asocia al poder de la adivinación, configurando una personalidad líder «todopoderosa» dentro de la comunidad. La cosmovisión de los negros e indios en esta región del Chocó, es el resultado del encuentro de tres mundos (indígena, hispánico y africano), que aun cuando sean muy heterogéneos por fuerza mayor de la historia, debieron encontrarse en el resultado de lo que hoy constituye la esencia cultural y mestiza de la identidad americana. Estas «cabezas médicas» son reconocidas por el desempeño especial de sus funciones. Así se les conoce como: brujos, teguas, chamanes, jaibanás, yerbateros, magos, leedores de la suerte, adivinos, chinangos, etc. Asimismo, las enfermedades y curas, las fórmulas, oraciones y secretos de curaciones y embrujamientos, hechizos y envenenamientos, maneras de invocar los espíritus, entrar en contacto con los muertos y en general todo el pensar, decir y actuar está regido por la «entrega» o «fe» en un espíritu «superior», que es el que «presta la ayuda» deseada para hacer el bien o el mal.

De la pléyade de «cabezas médicas» conocidas, muchos tuvieron que pasar o aprender de la mano de un «sabio» tradicional experimentado, ingresando a verdaderas escuelas tradicionales de adiestramiento, donde fueron sometidos a pruebas de identificación de enfermedades, plantas curativas y aplicación de recetas. Muchos tuvieron que pasar la prueba de la «tonga», a través de la ingestión de alucinógenos para poder «viajar» al interior del mundo de los espíritus. Además debieron aprender a leer orines, pulsear, contar el jai, luchar contra demonios y someterlos a su fiel voluntad, distinguir entre las plantas que sirven para curar y aquellas que sirven para atacar a sus contrarios o hacer el mal. Aprender de memoria un catálogo de oraciones para «tramar y destramar», poner y quitar tamboritos y un sinnúmero de exigencias cumplidas, hasta finalmente recibir los bastones de jaibaná, los que representan los espíritus familiares que su maestro entrega al aprendiz, para que «vaya por el mundo a cumplir su misión de médico, chamán, curandero, chinango o como este se quiera llamar» en la escatología del saber ancestral del mundo divino y del mundo humano.

\section{CONCLUSIONES}

Las «cabezas médicas» del Chocó biogeográfico y en especial las ubicadas en el municipio de Pizarro en el Baudó, representadas por indígenas y afrodescendientes, contribuyen a la conformación de una historia intercultural de enriquecimiento de los hispanoamericanos.

La etnomedicina o sistemas terapéuticos tradicionales del municipio de Pizarro se rigen por diversos paradigmas de salud que involucran el equilibrio psicológico-mágico-religioso, las enfermedades latentes inherentes a todos los integrantes de su comunidad y los elementos naturales del medio. En este sentido las «cabezas médicas» tanto afrodescendientes como indígenas han mantenido vivas sus tradiciones no solo como un mecanismo de conservación cultural sino como una estrategia para mejorar las condiciones de vida de su comunidad, que generalmente presenta bajos niveles de atención en salud.

Aunque las «cabezas médicas» afrodescendientes e indígenas presentan diferentes prácticas, dioses y denominaciones para sus actividades etnomedicinales, coinciden en el uso de los elementos de la naturaleza como principal materia prima para la elaboración de ritos, curas, riegos y amuletos entre otros.

La medicina tradicional afro e indígena ha sido la solución a la situación general de salud en el municipio de Pizarro que resulta ser de muy bajo nivel, debido entre otros factores al aislamiento geográfico y la consecuente dificultad para el sistema oficial de salud de proveer servicios de calidad a estas comunidades, lo que incluye las carencias económicas, así como de personal profesional y de infraestructuras adecuadas.

\section{LITERATURA CITADA}

Aprile-Gniset, J. 2004. Apuntes sobre el poblamiento del Pacífico. Panorámica afrocolombiana. p. 269-87. Bogotá: Centro de Estudios Sociales Universidad Nacional de Colombia, Instituto Colombiano de Antropología.

Friedemann, N. S., J. Arocha. 1995. Los diablos: de las iglesias al carnaval. p. 109-13. En: Diversidad es riqueza: ensayos sobre la realidad colombiana. 


\section{Conocimiento tradicional de los saberes médicos en Pizarro. JD. Mosquera}

Bogotá: Ican-Consejería Presidencial para los Derechos Humanos. Antón Sánchez, J. 1998. El conocimiento tradicional de las comunidades negras asociado a más prácticas mágico curativas de un área del Chocó. Tesis de Grado en Antropología, Bogotá: Universidad Nacional. 150 pp.

Binder, H., M. Cerán. 1971. La religiosidad del moreno en la costa caucana del Pacífico. Tesis de Filosofía. Bogotá: Universidad Tomás de Aquino. $165 \mathrm{pp}$.

Branislaw, M. 1994. Magia, ciencia y religión. Barcelona: Editorial Ariel. p. 1117-23.

Caicedo, M. A. 1997. Chocó mágico y folclórico. Quibdó: Ed. Gráficas Universitarias. p. 108

De Granda, G. 1977. Fórmulas mágicas de conjuros en el departamento del Chocó. Tomo XXII, $\mathrm{N}^{\circ}$ 1. Madrid: Thesaurus. p. 166-73.

Gutiérrez de Pineda, V. 1985. La medicina tradicional y curanderismo en Colombia. Tomos I y II. Bogotá: Universidad Nacional de Colombia. 143 pp.

Galindo Mendoza, G. 1994. Experiencias míticas y curaciones por medio del secreto en el Patía, Cauca. Tesis de grado. Popayán: Universidad del Cauca. 156 pp.

Escalante Polo, A. 1993. Aspectos mágico-religiosos presentes en la cultura popular de la costa atlántica de Colombia y sus posibles orígenes. En: Ulloa,
A. (Ed). Contribución africana a la cultura de las Américas. Bogotá: Instituto Colombiano de Antropología-Biopacífico. 125 pp.

Izquierdo, G. 1991. El mundo religioso del afroamericano del litoral Pacífico. Ensayo e interpretación. p. 96-110. En: Teológica Javeriana. Bogotá: Pontificia Universidad Javeriana.

Losonczy, A. 1933. De los vegetales a lo humano: un modelo cognitivo afrocolombiano del Pacífico. Rev Colomb Antropol XX: 37-58.

Lozano, L. A. 1987. La muerte en el departamento del Chocó. Rev CODECHOCO. 10: $20-32$.

Pollak Eltz, A. 1994. Religiones afroamericanas hoy. Caracas: Planeta. 268 pp. Velásquez Murillo, R. 1957. Muestras de fórmulas médicas utilizadas en el Alto y Bajo Baudó. Bogotá: Universidad Nacional de Colombia.89 pp.

Vasco, L. G. 1982. Jaibanás. Los verdaderos hombres. Bogotá: Banco Popular, Colección Textos Universitarios. 96 pp.

Reichel-Dormatoff, G. 1979. Conceptos indigenas de enfermedady de equilibrio ecológico: los Tukano y los Rogi de Colombia. p. 151-162. Simposio Internazionale sulla Medicina Indigena e Popolare dell'America Latina, Roma 1977. Roma: Instituto Italo/Latino Americano-ILA.

Herrera, X., M. Lobocuerrero. 1982. Antropología médica y medicina tradicional en Colombia. Temario guía y bibliografía anotada. Bogotá: Centro Cultural Jorge Elicécer Gaitán. 169 pp. 